\title{
Modelling and forecasting of electrical consumption for demand response applications
}

\author{
Iacob Crucianu, Otilia Bularca \\ SIVECO Romania SA \\ Bucharest, Romania \\ \{Iacob.Crucianu, Otilia.Bularca\}@siveco.ro
}

\author{
Ana-Maria Dumitrescu, \\ Politehnica University of Bucharest \\ Bucharest, Romania \\ Anamaria.Dumitrescu@upb.ro
}

\begin{abstract}
The emergence of concepts, policies and applications like smart grid, energy communities, carbon footprint reduction, energy-efficient consumption, demand response, demand side management enables the development and implementation of algorithms and procedures dedicated to the analysis of domestic energy consumption. In this paper a method of modelling and forecasting is presented and an optimization of electric energy consumption using machine learning and a cost function based on local peaks identified in load curves is proposed.
\end{abstract}

Index Terms - optimization, modelling, forecasting, demand response, electric energy consumption, machine learning

\section{INTRODUCTION}

Global electric energy consumption has increased significantly and continuously since 1960s in all energy sectors, including residential usage (Fig. 1), [1]. In 2014 residential sector amounted to $25 \%$ of the overall EU-28 electric energy consumption, while the consumption in all buildings represented about $40 \%$ [2]. Domestic consumer has a key role in the energy consumption value chain and energy management approaches are drivers for new technologies and innovation [3]. The focus of these approaches is not only on technical and economical factors, but also on awareness, engagement and identifying the possibilities of incentivizing the Consumer.

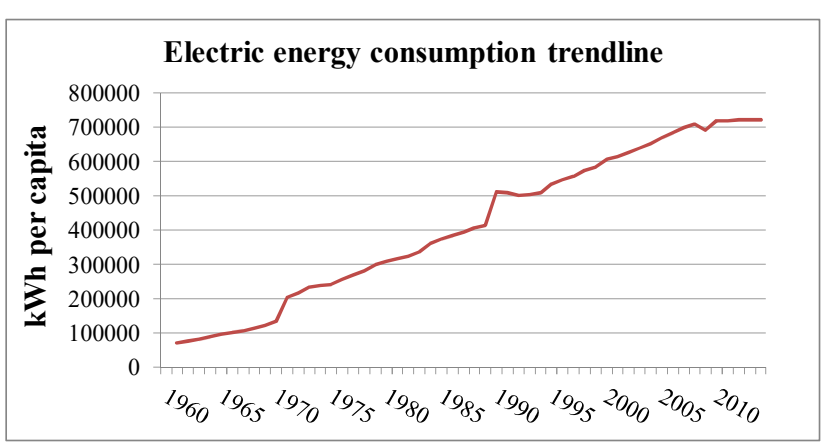

Figure 1. Electric power consumption (kWh per capita) [1]

To some extend the technology society seems to approach its limits and technological progress is no longer selfsufficient in the context of energy efficiency. Proposed solutions include prosumer engagement, demand response, demand side management, building energy management systems and other optimizations [4].

Consumers may play a significant role in day by day operation of electric (smart) grid by reducing or shifting their electricity usage during peak periods in response to dynamic tariffs or incentives. This can be done by locally producing part of the necessary energy (prosumers), installing local storage or engaging in demand response. Demand response is a demand-side resource that enables consumption optimization based on prior agreements between energy market actors (DSOs, supplier, aggregators, end-consumers). The core of dedicated DR applications is based on concepts like cloud computing, machine learning, artificial intelligence and big data.

The paper describes one of these demand response algorithms including data storage, peak detection and possible mitigation plus a forecasting approach both from Customer and DSO point of view. The main difference between the two perspectives is related to the point of measurement and level of aggregation, since for the DSO the information is stored for all consumers/customers. The intervals for the time aggregation are the same for consumers and DSO (15' or less, one hour, one day, one week, one month and one year).

The approach described in this paper is based on a supervised, model-based Machine Learning system that uses Linear Regression Algorithm based on spline curves and is able to learn batch volumes of data including online data incrementally stored. The system is used to detect peak values, to propose optimized behaviour and forecasting and to run what-if scenarios. The input information consists in metering data collected for at least two years.

The study is based on a $\mathrm{H} 2020$ project that demonstrates a number of demand response activities including one in Ploiesti, Romania. As part o this activity consumption patterns are identified and optimization tasks are proposed based on forecasting data and applied criteria.

The rest of the paper is structured in four main sections. Section two describes the general principles of machine learning that are implemented in this research. Sections three 
and four describe the optimization and forecasting proposed algorithms accompanied by numerical simulation results in section five.

\section{MACHINE LEARNING}

Machine learning is an artificial intelligence application which uses available computers' power to implement learning mechanisms. Machine learning algorithms are broadly classified into three types: i) supervised learning; ii) unsupervised learning; and iii) reinforcement learning [5].

The evolution of machine learning is comparable to that of data mining, i.e. both consider or explore from end to end data to search for patterns. On the other hand, while data mining applications extract information for human knowledge, machine learning generally uses the data to identify patterns and fine-tune program actions.

Machine learning [6] offers great opportunity for systems meeting at least one of the following characteristics:

- the problem has many parameters or rules;

- large amounts of data is available and needs analysis;

- the problems are complex and there is no general solution;

- the environments are fluctuating, and the solution needs to be adapted.

Previous information matched Ploiesti Pilot with all of these four characteristics mentioned above. Thus, for implementing demand response optimization an approach using machine learning was proposed and when choosing the type of machine learning system some facts were considered based on the particularities of the pilot case:

- the system should be trained with human supervision;

- the system can be trained both in batch mode and incrementally during operation;

- the system should detect patterns in the training data and build predictive models.

Consequently, a supervised learning system using a linear regression algorithm [7] able to learn data in batch volumes and also incrementally was selected. A model-based learning approach is used considering that the system should detect patterns and based on the type of available data. The preferred models are a polynomial model for short time intervals and splines based on cube polynomials (natural cubic splines) for longer time intervals (months, years). The formulae used depending on the situation are cubic polynomials of the form:

$$
\mathrm{P}(\mathrm{x})=\sum_{\mathrm{i}=0}^{3} \mathrm{a}_{\mathrm{i}} \mathrm{x}^{\mathrm{i}}
$$

while the polynomial for a specific time interval, considered between two peaks is:

$$
P_{i}(x)=\sum_{k=0}^{3} a_{k}\left(x-x_{i}\right)^{k}
$$

where $i$ is the number of the peak $(>1)$.

The border of the spline is defined by the peak values detected using conditions first and second derivative.

\section{OPTIMIZATION}

\section{A. Teoretical approach}

The optimization algorithm proposed in this study is based on peak values and its goal is to maintain the consumption level without affecting the comfort level of the customer while decreasing the consumption during peak times and respectively increasing it in periods with low consumption. To implement this gradient descent [8] optimization algorithm with a cost function based on mean square error is used.

Since the correlation between elements of multidimensional values is usually very low the computations were initiated based on "distance correlation" [9]. For instance, the correlation between active power and down time is low (near 0). Consequently, instead of working with one time series and multidimensional (n dimensional) values, $n$ time series with one dimensional data are treated separately.

It was observed that the histogram of data registered for many customers, for a specific period of time, follows a distribution governed by Kumaraswamy's [10], double bounded distribution. The probability density function of the Kumaraswamy distribution is

$$
f(x ; a, b)=a b x^{a-1}\left(1-x^{a}\right)^{b-1}
$$

where $\mathrm{x} \in[0,1]$ and $a, b$ are non-negative shape parameters.

The histogram contains on the $x$ axis a value range and on the $y$ axis the number of customers with the value of the studied parameters in the specified range.

For the distribution form and the parameters the following formula was implemented:

$$
\operatorname{RMSE}(\mathbf{X}, \mathrm{h})=\sqrt{\frac{1}{\mathrm{~m}} \sum_{\mathrm{i}=1}^{\mathrm{m}}\left(\mathrm{h}\left(\mathrm{x}^{\mathrm{i}}\right)-\mathrm{y}^{\mathrm{i}}\right)^{2}} .
$$

The tool is calibrated using the values $a$ in interval $a \in[3$, $4]$, and $b$ on interval $b \in[4,5]$. The calibration was performed by analyzing input data using clustering algorithms and the values were placed in (4).

\section{B. Training, Testing and Validation}

The training, testing and validation were done following data domains presented below:

- Data registered in a day cumulated at hour level

- Data registered in a week cumulated at day level

- Data registered in a month cumulated at day level

- Data registered in an year cumulated at month level

- The data considered is divided in:

- Data for consumers

- Data for DSO

For each detailed level the data registered in two years related to power consumption were considered and divided in three parts: 
- $80 \%$ of data was training data

- $10 \%$ of data was testing data

- $10 \%$ of data was validation data.

The performance measure of the system is Root Mean Square Error

$$
\operatorname{RMSE}(\mathrm{X}, \mathrm{h})=\sqrt{\frac{1}{\mathrm{~m}} \sum_{\mathrm{i}=1}^{\mathrm{m}}\left(\mathrm{h}\left(\mathrm{x}^{\mathrm{i}}\right)-\mathrm{y}^{\mathrm{i}}\right)^{2}}
$$

where

$$
\begin{aligned}
& m: \text { is the number of instances } \\
& x^{i} \text { is the vector of values } \\
& y^{i} \text { is the vector of desired values } \\
& h(x) \text { is the prediction function }
\end{aligned}
$$

$X$ is a matrix containing all the feature values

Mean absolute error is used to increase the speed of processing and the model is adjusted during testing to minimize the generalization error.

The model presented in this section considers no disturbances in the system. Disturbances were included in the forecasting and what-if scenarios and are in the form datetime, duration, sign, and percentage. Disturbances cover: weather conditions, national and other holidays, and special conditions.

The objectives of the proposed optimization algorithm include:

- detection of the peak time intervals and the peak values;

- optimizing the power consumption and providing suggestions to DSO and customers;

- $\quad$ proposing optimizations for demand response system;

- forecasting with or without disturbances;

- simulation of what-if scenarios.

Two types of peak values are considered:

Positive peak - a value where there is a change in the time series gradient from positive to negative. The peak is considered only if before the change of gradient from positive to negative there were at least $n$ positive gradients and $n$ negative gradients ( $n>1$, usually $n=4)$;

Negative peak - a value where there is a change in the time series gradient from negative to positive. The peak is considered only if before the change of gradient from negative to positive there were at least $n$ negative gradients and $n$ positive gradients ( $n>1$, usually $n=4)$.

Peak detection is performed on all data stored from smart meters, but peaks are identified inside dedicated time intervals as local peaks (e.g. peak hours per day) and not as global peaks.

Peak time values are identified considering:
- $\quad$ maximum or minimum values registered in a specified time-period;

- gradient of consumption changes in a period of time.

It is worth mentioning that the number of retrieved peaks can be preset at the begging of the process.

Fig. 2 presents a test case where the number of peaks is $n=2$. The principles of optimizations used by the system are:

- the positive peaks are decreased with a value dependant on the gradient before and after the peak;

- the negative peaks are increased with a value dependant on the gradient before and after the peak;

- the sum of the values for the data domain selected remain the same.

The chart presents an optimized time series based on the peak registered values highlighting the load curve before and after optimization.

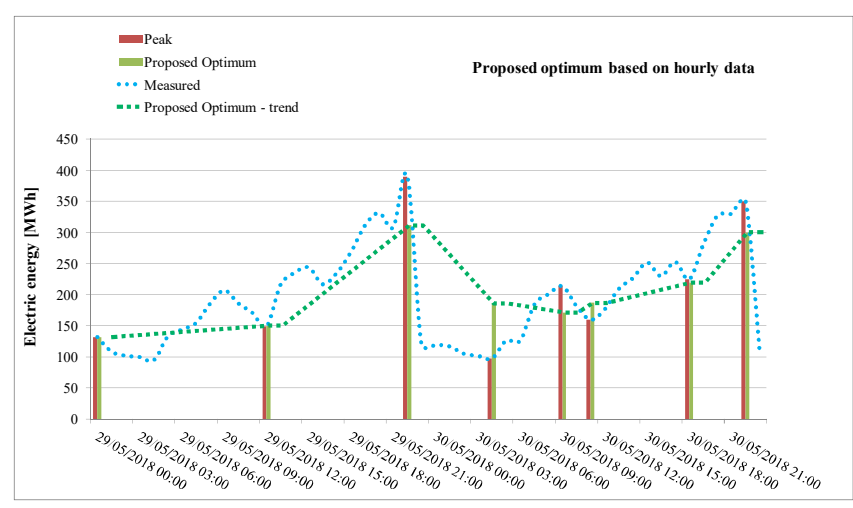

Figure 2. Optimized raw time series

This procedure is implemented as part of demand response applications based on forecasted data.

\section{CONSUMPTION FORECASTING}

\section{A. Forecasting without external perturbations}

An important part of implementing demand response algorithms is the generation of suggestions for end-consumers. For this different scenarios need to be simulated. These scenarios are based on prior consumption behaviour, energy generation provisions, other constraints and forecasted consumption and generation curves based on these data.

Monte Carlo simulation and a specific statistical distribution are usually used for forecasting [11], [12]. To improve the accuracy in this study machine learning principles are applied due to the big set of historical data available.

In this study forecasting is performed on all data stored from smart meters and uses machine learning systems to get the regression line. For this purpose supervised learning with batch data collected for last two years is considered. The resulting regression line is adjusted by online learning of currently available data [13]. 
The algorithm also optimizes the predicted consumption and the output is a polynomial regression curve.

The regression model proposed is based on polynomials (cube polynomials) and spline functions. Forecasted data is calculated for a reference dataset.

Based on the input reference date a similar period of time is found and similar periods of time are selected depending on the data model.

To forecast quantities aggregated at one hour level for one specific day (in the future) similar day(s) in the dataset is selected based on different criteria. For instance, if the reference date is 10th of October 2019, then the similar period is 11 th of October 2018 because

- $\quad$ the system is trained with data from 2018;

- $\quad$ October is the month to be forecasted;

- 10th October 2019 is Thursday and 11th October 2018 is the closest Thursday.

Similar rules apply to day, week and month cumulated data.

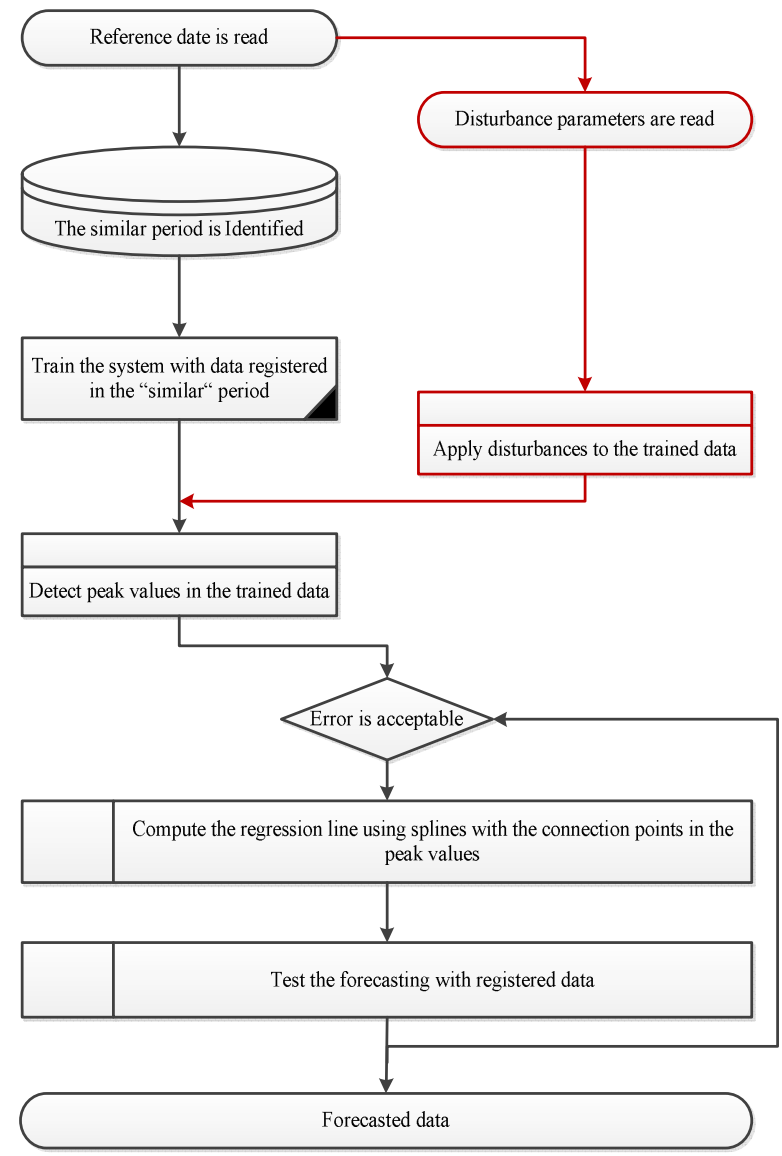

Figure 3. Forecasting algorithm

The forecasting algorithm is described in Fig.3.

\section{B. Forecasting with disturbances}

In this study what-if scenarios are defined as forecasting with disturbances. The same element used in forecasting applies to what-if scenarios, but the algorithm is different. This forecasting uses the same machine learning algorithm like the previous one (no external perturbations). But after applying the model trained for forecasting, the points which are defining the regression line will be changed according with values manually registered as perturbations. What-if scenario algorithm is also described in Fig. 3 and the impact of disturbances is highlighted in red. The process is repeated starting with step 2 (disturbance parameters are read) until all requested scenarios are simulated.

\section{NumERICAL Simulation}

For the data collected in last two years different time intervals to analyze data were used: one hour, one day, one week, one month, and one year. Based on these time intervals it was possible to create data models for the evolution of consumption parameters at different detail level. Averages and standard deviations are calculated and represented in Fig 4.

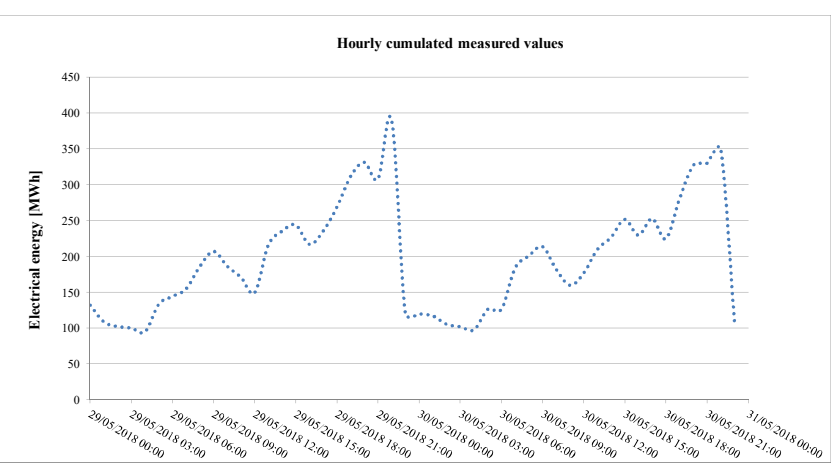

a)
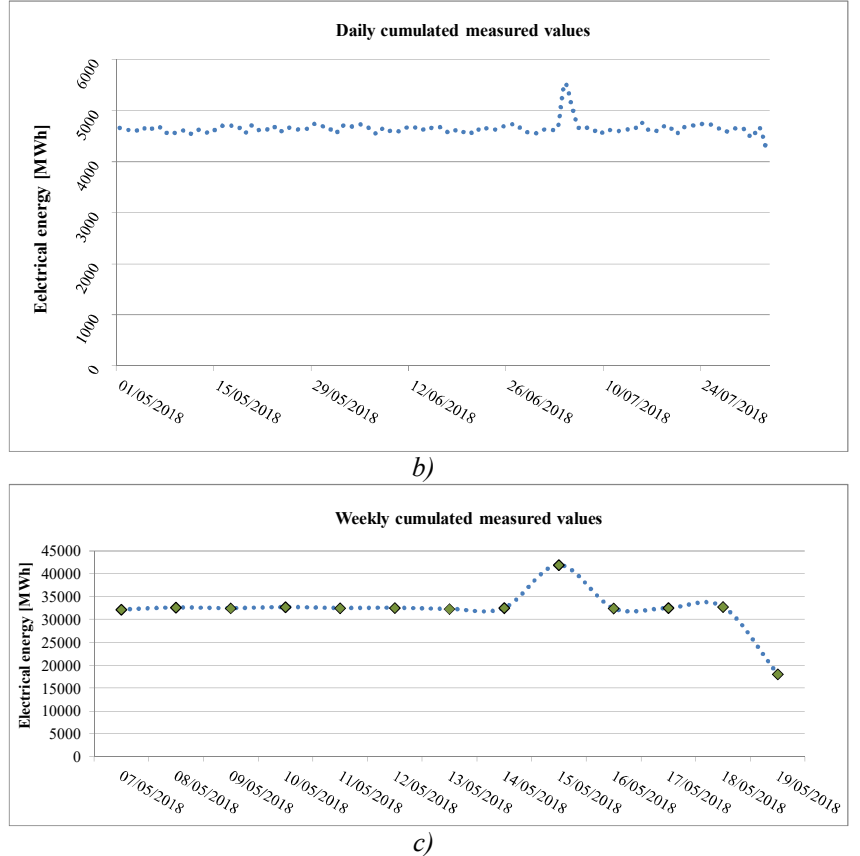

Figure 4. Collected data - a) hourly agreggation, b) daily agreggation, c) weekly agreggation 
The data considered represent time series of values. All parameters registered so far, or all parameters expected to be registered as values coming from smart meters are in the category of time series. For the time series considered interpolation, optimizations and predictions (forecasting) was performed.

The time series refers to multidimensional values. A multidimensional value is composed by several parameters registered by smart meters. Several parameters are considered for the optimization or forecasting in this study: $\{P, Q, U, U p$ and Down Time, SAIDI\}.

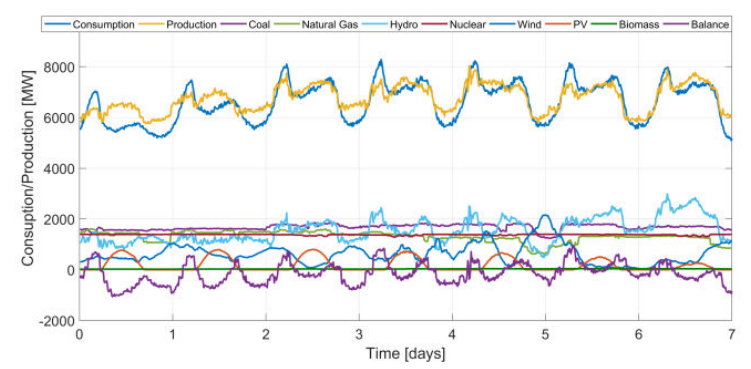

a)

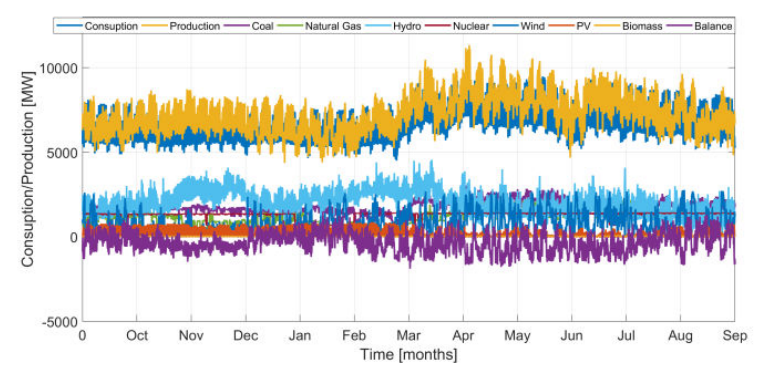

b)

Figure 5. Evolution of consumption a) one week, b) in one year [14]

In Fig. 5 data for years 2017 and 2018 are represented for different time intervals.

Based on the two year dataset and the samples presented in Fig. 4 and 5 a sensitive analysis was performed with the following outcomes:

- In one day the evolution is dependent on hours. There are peak values, and the study of data is very important to understand the system, and next to optimize it or to create forecasts.

- In one week there are different values in working days than in weekend. It is important to study the evolution of data for one week, to be able to propose recommendations and to make forecasts.

- In one month the evolution is following the weekly evolution. There are no important differences between working days in different period of month, but there are differences between working and not working days.

- In one year there is an evolution on monthly basis. In some month the consumption is bigger than in others.
Even though there is a global trend, for monthly evolution the standard deviation is bigger than for other period of time.

In Fig. 6 day by day forecast for electrical energy consumption is presented: blue represents the real consumption and red represents the forecasted optimum line. Similarly, forecasting is performed for weeks, months, years.

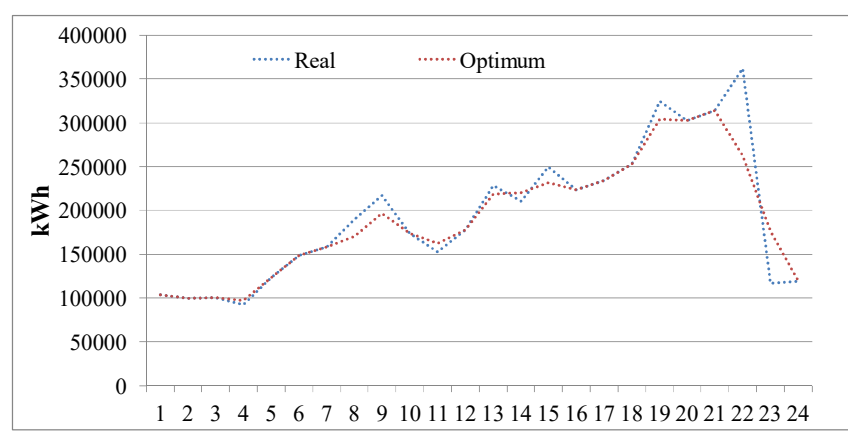

Figure 6. Forecasting - no external perturbations

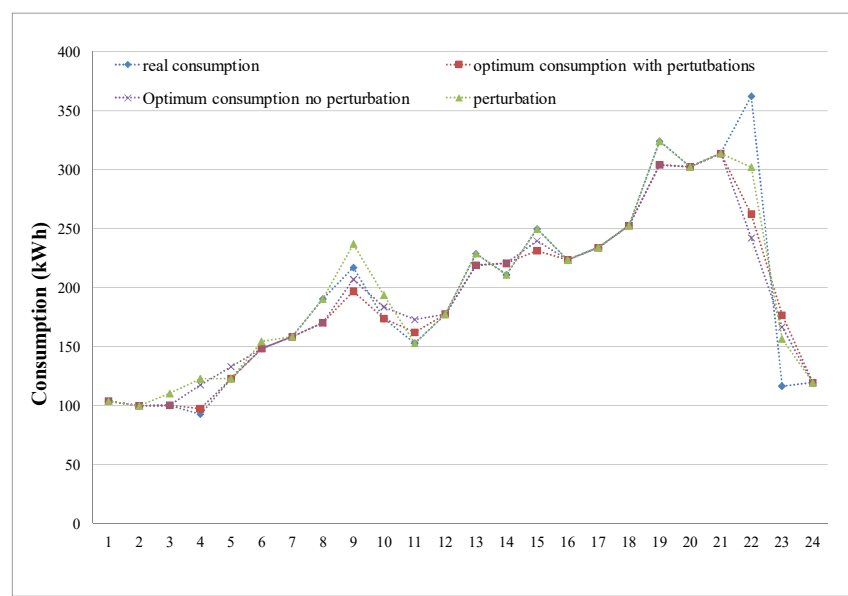

Figure 7. Forecasting -with and without external perturbations

In Fig. 7 day by day forecast with disturbances for electrical energy consumption is presented. In this case the points marked in red can be forced to take other values based on weather conditions, or other external events.

\section{CONCLUSIONS}

Classical methods of matching the demand for power with the supply rely on adjusting the supply (by turning on and off different generators). On the other hand demand response engages the consumers in adapting their own consumption behaviour and aims at changing their habits on long term. This can be done sending suggestions to consumers to improve their load curve.

Suggestions sent by the DSO are issued by applications using smart algorithms that implement machine learning optimization techniques.

In this paper a method of modelling and forecasting is presented and an optimization of electric energy consumption using machine learning and a cost function based on local peaks identified in load curves is proposed. 
The optimization algorithm presented was chosen considering the amount of available data, real-time response interval and the mathematical models that may describe the consumption pattern for a large number of different consumers. While more mathematically elaborate algorithms could provide a better optimization for particular cases they need more resources (processing power and expertise) and tend to provide bespoke solutions that might not be suitable for large scale applications with uncorrelated inputs. Moreover, the deployment of such an application relies on suggestions for long term improvement and continuous adjustments based on previous data and don't aim at an absolute mathematical optimum.

All the algorithms discussed in this document are applied to the time intervals presented and will be implemented and further validated in Ploiesti pilot site involving both DSO and real consumers.

\section{ACKNOWLEDGMENT}

The project leading to this application has received funding from the European Union's Horizon 2020 research and innovation programme under grant agreement No 731268.

\section{REFERENCES}

[1] Electric power consumption (kWh per capita), Database available at: https://data.worldbank.org/indicator/EG.USE.ELEC.KH.PC, accessed on 14th of December 2018

[2] Clare Ferguson, Marc Hall, Energy efficiency in buildings, European Parliamentary Research Service Blog, July 8, 2016

[3] Raka Jovanovic, Abdelkader Bousselham and Islam Safak Bayram, Residential Demand Response Scheduling with Consideration of Consumer Preferences, Applied Sciences, Volume 6, Issue 1, 2016, doi:10.3390/app6010016
[4] Soumya Kundu, Thiagarajan Ramachandran, Yan Chen and Draguna Vrabie, Optimal Energy Consumption Forecast for Grid Responsive Buildings: A Sensitivity Analysis, 2018 IEEE Conference on Control Technology and Applications (CCTA), 21-24 Aug. 2018, Copenhagen, Denmark

[5] Chenn-Jung Huang ; Ming-Chou Liu ; San-Shine Chu ; Chin-Lun Cheng, Application of machine learning techniques to Web-based intelligent learning diagnosis system, Fourth International Conference on Hybrid Intelligent Systems (HIS'04), 5-8 Dec. 2004, Kitakyushu, Japan.

[6] A. Geron, in Hands-On machine Learning with Dcikit-Learn \& TensorFlow, O'Reilly, 2017

[7] J.E. Dennis Jr., R.B. Schnabel, "Numerical methods for unconstrained optimization and nonlinear equations" , Prentice-Hall, 1983

[8] Jan A Snyman, Daniel N Wilke, Practical Mathematical Optimization, Basic Optimization Theory and Gradient-Based Algorithms, Springer, 2018

[9] G. J. Szekely and M. L. Rizzo, Energy statistics: A class of statistics based on distances, Journal of Statistical Planning and Inference, Volume 143, Issue 8, August 2013, Pages 1249-1272

[10] M.C.Jones, Kumaraswamy's distribution: A beta-type distribution with some tractability advantages, Statistical Methodology, Volume 6, Issue 1, January 2009, Pages 70-81

[11] Bansal, A., Rompikuntla, S.K., Gopinadhan, J., Kaur, A., Kazi, Z.A.: Energy consumption forecasting for smart meters. arXiv preprint arXiv: $1512.05979,2015$

[12] Khuram Pervez Amber 1, Muhammad Waqar Aslam, Anzar Mahmood, Anila Kousar, Muhammad Yamin Younis, Bilal Akbar, Ghulam Qadar Chaudhary and Syed Kashif Hussain, Energy Consumption Forecasting for University Sector Buildings, Energies 2017, vol. 10, issue 10

[13] Rodrigo F. Berriel, Andre Teixeira Lopes, Alexandre Rodrigues, Flavio Miguel Varej ' ao, Thiago Oliveira-Santos, Monthly Energy Consumption Forecast: A Deep Learning Approach, International Joint Conference on Neural Networks (IJCNN), 14-19 May 2017, Anchorage, AK, USA

[14] Generation and consumption levels rerported by Romanian TSO, available at: http://www.transelectrica.ro/widget/web/tel/sen-grafic//SENGrafic_WAR_SENGraficportlet?display=APE 